\title{
Attenuation of Seismic Energy in the Upper Mantle ${ }^{1}$
}

\author{
Don L. Anderson, Ari Ben-Menahem, and C. B. Archambeat ${ }^{2}$ \\ Seismological Laboratory \\ California Institute of Technology, Pasadena
}

\begin{abstract}
The amplitude attenuation and phase dispersion for Love and Rayleigh waves in the period range 50 to $300 \mathrm{sec}$ is determined from two earthquakes by digital techniques. A distribution of $Q$, or anelasticity, is determined for the upper mantle which satisfies the amplitude decay data for Love and Rayleigh waves and which is consistent with available body wave data. An argument is made for a longitudinal wave $Q$ of about 2.4 to 2.6 times the $Q$ for shear waves. This implies that very small losses are involved in pure compression compared to the losses in shear. This is an argument against the importance of certain mechanisms, such as thermoelastic losses, in the mantle. The $Q$ for shear waves in the upper $400 \mathrm{~km}$ of the mantle seems to vary from about 50 to about 150 . The $Q$ for mantle Rayleigh waves is greater than the $Q$ for mantle Love waves, both theoretically and experimentally. However, it is predicted that $Q_{R}$ becomes less than $Q_{L}$ at some period shorter than 50 sec, the crossover period being diagnostic of the thickness of the ' $Q$ crust' or lithosphere.
\end{abstract}

Introduction. In most discussions of the properties of the earth's interior the elastic properties determined by seismological techniques are used directly or indirectly. The velocity of transport of seismic energy has been the principal measured parameter in theories related to the density distribution, thermal properties, composition, and phase of the earth's interior. A complete description of the energy transport phenomenon involves the rate of energy dissipation as well as the velocity of propagation. In modem theoretical and experimental work on the physics of solids both parameters are important. In general, the rate of energy dissipation, or anelasticity; is a more sensitive indicator of changes in crystalline structure due to temperature and phase change than the elasticity is. It is also a more pertinent quantity for many geologic problems. Unfortunately, accurate amplitude information is more difficult to obtain and interpret than travel-time data. Instrumental effects, local geology, mode conversion, scattering, and source characteristics have tended to obscure the amplitude variations due to true energy dissipation.

${ }^{1}$ Contribution 1292, Division of Geological Sciences, California Institute of Technology, Pasadena.

"Now at Tnited Earth Scioness, Alexandria, Virginia.
Amplitudes of body waves are particularly difficult to interpret because of mode conversion at interfaces, scattering, and complicated spreading losses.

A technique has recently been developed for the interpretation of frequency-dependent amplitude decay data for surface waves in terms of anelasticity versus depth [Anderson and Archambeau, 1964]. The advantages of the surface wave method for studying attenuation are:

1. The Iong-period waves suffer less degradation due to inhomogeneities, i.e. Rayleigh-type scattering.

2. A given bundle of energy can be sampled at several points along its own ray, i.e. the surface of the earth.

3. The geometric spreading factor can be calculated more accurately.

4. Velocity reversals and regions of the mantle where body waves suffer anomalous refraction such as first-or second-order velocity discontinuities offer no particular difficulty.

5. Successive passages of longer-period surface waves from large earthquakes past a given instrument can be analyzed, removing completely the effects of source asymmetry, instrumental effects, local geology, and geometric spreading.

6. A single seismogram contains sufficient information for a fairly detailed interpretation of structures. 
We Fourier analyzed strain records of the Chile earthquake of May 22, 1960, and pendulum records of the Iran shock of September 1, 1962 , to determine the phase and group velocities and the rate of decay of Love and Rayleigh wave energy in the spectral band of 50 to 300 sec. These data are used to determine the attenuation in the upper mantle.

Data analysis. For periods between 150 and 333 sec we utilized the waves $R_{2}, R_{4}, G_{2}$, and $G_{4}$ from the Chile earthquake recorded at Pasadena on the Benioff NS strain seismograph. All data were digitized at 3 - or 4 -sec spacings, digitally filtered and Fourier analyzed by conventional techniques [see Alexander, 1963; Toksöz and Ben-Menahem, 1963]. Phase velocities $c$ were computed from the pairs $R_{4}-R_{2}$ and $G_{4}-G_{2}$, and group velocities $U$ were obtained from these data by first-order differences. The absorption coefficients $k^{*}$ were computed from the spectral amplitude ratios of these pairs. The quintity $Q$ was computed from

$$
Q=\pi / k^{*} U T
$$

For periods between 50 and 125 sec we used data from the Toledo and Trinidad stations of the World Wide Network of Standardized Seismographs stations for the Iran shock. The wave trains $R_{1}$ and $G_{1}$ were digitized at 2-sec intervals and analyzed as above. The resulting velocity and attenuation data are appropriate for a primarily oceanic path. Tables 1 and 2 give the resulting propagation parameters. The shorterperiod data are less certain than the longerperiod data because of the corrections necessary for station orientation and spreading, and the slight departure from a true great-circle path which was ignored. Note that $Q_{R}$ is about $13 \%$ greater than $Q_{r}$ at $50 \mathrm{sec}$ and increases to about $30 \%$ greater at 200 sec. At 300 sec $Q_{R}$ is almost $70 \%$ greater than $Q_{L}$.

Theory of attenuation. The phase amplitude changes of a propagating body wave can be represented by a complex wave number or propagation constant,

$$
\mathbf{k}=k+i k^{*}
$$

or a complex velocity

$$
\boldsymbol{\alpha}=\alpha-i \alpha^{*}=\omega / \mathbf{k}_{\alpha}
$$

for compressional waves of period (a) and

$$
\boldsymbol{\beta}=\beta-i \beta^{*}=\omega / \mathbf{k}_{\beta}
$$

\begin{tabular}{|c|c|c|c|c|c|c|c|c|c|c|c|c|}
\hline \multirow[b]{2}{*}{$\begin{array}{c}f, \\
\mathrm{mc} / \mathrm{s}\end{array}$} & \multirow[b]{2}{*}{$\begin{array}{l}T, \\
\text { sec }\end{array}$} & \multicolumn{5}{|c|}{ Rayleigh Waves } & \multicolumn{5}{|c|}{ Love Waves } & \multirow[b]{2}{*}{$Q_{R} / Q_{L}$} \\
\hline & & $\begin{array}{l}2 \pi h^{-1} \\
\mathrm{~km}\end{array}$ & $\begin{array}{c}{ }^{\prime}{ }_{R}, \\
\mathrm{~km} / \mathrm{sec}\end{array}$ & $\begin{array}{c}U_{R}, \\
\mathrm{~km} / \mathrm{sec}\end{array}$ & $\begin{array}{c}k^{*} \\
\mathrm{~km}^{-1}\end{array}$ & $Q_{k}$ & $\begin{array}{c}2 \pi k^{-1} \\
\mathrm{~km}\end{array}$ & $\begin{array}{c}{ }^{\prime} L \\
\mathrm{~km} / \mathrm{sec}\end{array}$ & $\begin{array}{c}U_{L}, \\
\mathrm{~km} / \mathrm{sec}\end{array}$ & $\begin{array}{l}k^{*} \\
\mathrm{~km}^{-1}\end{array}$ & $Q_{L}$ & \\
\hline 3.0 & 333.3 & 1894 & 5.548 & 3.95 & $0.9 \times 10^{-5}$ & 265 & 1769 & 5.307 & 4.44 & $1.6 \times 10^{-5}$ & 135 & 1.96 \\
\hline 3.2 & 312.5 & 1682 & 5.383 & 3.80 & 1.1 & 240 & 1638 & 5.243 & 4.42 & 1.7 & 135 & 1.78 \\
\hline 3.4 & 294.1 & 1545 & 5.251 & 3.70 & 1.3 & 222 & 1525 & 5.185 & 4.40 & 1.8 & 135 & 1.64 \\
\hline 3.6 & 277.8 & 1426 & 5.132 & 3.61 & 1.4 & 223 & 1426 & 5.134 & 4.39 & 1.9 & 132 & 1.69 \\
\hline 3.8 & 263.2 & 1319 & 5.012 & 3.57 & 1.6 & 209 & 1339 & 5.088 & 4.38 & 2.1 & 130 & 1.61 \\
\hline 4.0 & 250.0 & 1229 & 4.917 & 3.56 & 1.8 & 196 & 1262 & 5.046 & 4.36 & 2.2 & 131 & 1.50 \\
\hline 4.2 & 238.1 & 1149 & 4.824 & 3.56 & 1.9 & 195 & 1192 & 5.008 & 4.33 & 2.3 & 132 & 1.48 \\
\hline 4.4 & 227.3 & 1081 & 4.754 & 3.53 & 2.1 & 186 & 1130 & 4.972 & 4.33 & 2.4 & 133 & 1.40 \\
\hline 4.6 & 217.4 & 1016 & 4.674 & 3.55 & 2.2 & 184 & 1074 & 4.940 & 4.32 & 2.5 & 131 & 1.41 \\
\hline 4.8 & 208.3 & 962 & 4.616 & 3.55 & 2.4 & 177 & 1023 & 4.911 & 4.32 & 2.6 & 131 & 1.35 \\
\hline 5.0 & 200.0 & 914 & 4.569 & 3.56 & 2.5 & 176 & 977 & 4.885 & 4.34 & 2.7 & 131 & 1.34 \\
\hline 5.2 & 192.3 & 869 & 4.517 & 3.58 & 2.7 & 169 & 935 & 4.861 & 4.34 & 2.9 & 131 & 1.29 \\
\hline 5.4 & 185.2 & 828 & 4.469 & 3.59 & 2.8 & 169 & 896 & 4.840 & 4.36 & 3.0 & 130 & 1.30 \\
\hline 5.6 & 178.6 & 792 & 4.436 & 3.61 & 2.9 & 168 & 861 & 4.821 & 4.36 & 3.1 & 130 & 1.29 \\
\hline 5.8 & 172.4 & 760 & 4.407 & 3.63 & 3.0 & 167 & 828 & 4.805 & 4.37 & 3.2 & 130 & 1.29 \\
\hline 6.0 & 166.7 & 729 & 4.374 & 3.65 & 3.1 & 16 & 798 & 4.790 & 4.37 & 3.3 & 130 & 1.28 \\
\hline 6.2 & 161.3 & 701 & 4.348 & 3.67 & 3.2 & 166 & 770 & 4.776 & 4.38 & 3.5 & 127 & 1.31 \\
\hline 6.4 & 156.2 & 675 & 4.319 & 3.70 & 3.3 & 164 & 744 & 4.761 & 4.38 & 3.6 & 127 & 1.29 \\
\hline 6.6 & 151.5 & 651 & 4.299 & 3.72 & 3.4 & 164 & 719 & 4.748 & 4.39 & 3.7 & 127 & 1.29 \\
\hline
\end{tabular}

for shear waves. The real parts of (3) and (4) are the intrinsic body velocities. A convenient

TABLE 1. Propagation Parameters, Chile-Pasadena Great Circle 
TABLE 2. Propagation Parameters, Toledo-Trinidad

\section{Rayleigh Waves}

f. $\quad T, \quad 2 \pi k^{-1}, \quad c_{R}, \quad U_{R}, \quad k^{*}$, $\mathrm{mc} / \mathrm{s}$ sec $\mathrm{km} \mathrm{km} / \mathrm{sec} \mathrm{km} / \mathrm{sec} \mathrm{km}^{-1}$

\section{Love Waves}

$2 \pi k^{-1}, \quad c_{L}, \quad U_{L}, \quad k^{*}$,

$\mathrm{km} \quad \mathrm{km} / \mathrm{sec} \mathrm{km} / \mathrm{sec} \quad \mathrm{km}^{-1} \quad Q_{L} \quad Q_{R} / Q_{L}$

\begin{tabular}{|c|c|c|c|c|c|c|c|c|c|c|c|c|}
\hline 8.0 & 125.0 & 586 & 4.250 & 3.70 & $5 \times 10^{-}$ & 151 & 586 & 4.690 & 4.35 & $5.8 \times 10$ & ${ }^{5} 109$ & 1.38 \\
\hline 8.8 & 113.6 & 477 & 4.196 & 3.72 & 4.7 & 158 & 531 & 4.673 & 4.35 & 5.8 & 109 & 1.45 \\
\hline 9.6 & 104.2 & 435 & 4.173 & 3.77 & 5.0 & 160 & 486 & 4.662 & 4.35 & 6.1 & 114 & 1.40 \\
\hline 10.4 & 96.2 & 401 & 4.167 & 3.82 & 5.6 & 152 & 447 & 4.652 & 4.35 & 6.2 & 121. & 1.25 \\
\hline 11.2 & 89.3 & 371 & 4.159 & 3.86 & 6.3 & 145 & 414 & 4.640 & 4.35 & 6.3 & 128 & 1.13 \\
\hline 12.0 & 83.3 & 347 & 4.167 & 3.90 & 7.0 & 138 & 386 & 4.627 & 4.35 & 6.7 & 129 & 1.07 \\
\hline 12.8 & 78.1 & 326 & 4.169 & 3.98 & 7.5 & 134 & 360 & 4.613 & 4.35 & 7.4 & 125 & 1.07 \\
\hline 13.6 & 73.5 & 306 & 4.160 & 4.04 & 8.0 & 132 & 338 & 4.603 & 4.34 & 8.8 & 112 & 1.18 \\
\hline 14.4 & 69.4 & 290 & 4.170 & 4.08 & 8.5 & 130 & 319 & 4.595 & 4.34 & 9.8 & 106 & 1.23 \\
\hline 15.2 & 65.8 & 274 & 4.163 & 4.10 & 9.2 & 127 & 302 & 4.588 & 4.33 & 10.9 & 101 & 1.26 \\
\hline 16.0 & 62.5 & 260 & 4.159 & 4.09 & 10.0 & 123 & 286 & 4.577 & 4.33 & 11.8 & 100 & 1.23 \\
\hline 16.8 & 59.5 & 247 & 4.155 & 4.07 & 10.9 & 119 & 271 & 4.558 & 4.32 & 12.3 & 100 & 1.19 \\
\hline 17.6 & 56.8 & 236 & 4.150 & 4.06 & 11.5 & 118 & 258 & 4.539 & 4.31 & 12.7 & 101 & 1.17 \\
\hline 18.4 & 54.3 & 225 & 4.145 & 4.05 & 12.0 & 119 & 246 & 4.522 & 4.29 & 13.0 & 103 & 1.15 \\
\hline 19.2 & 52.1 & 216 & 4.141 & 4.04 & 12.3 & 121 & 235 & 4.519 & 4.28 & 13.1 & 107 & 1.13 \\
\hline 20.0 & 50.0 & 207 & 4.136 & 4.04 & 12.4 & 125 & 225 & 4.498 & 4.27 & 13.2 & 111 & 1.13 \\
\hline
\end{tabular}

dimensionless measure of attenuation, $Q^{-1}$, is defined as the ratio of the energy dissipated per wavelength to the total energy carried, or

$$
\begin{aligned}
& Q_{\alpha}{ }^{-1}=2 k_{\alpha}{ }^{*} / k_{\alpha}=2 \alpha^{*} / \alpha \\
& Q_{\beta}{ }^{-1}=2 k_{\beta}{ }^{*} / k_{\beta}=2 \beta^{*} / \beta
\end{aligned}
$$

In terms of $Q$ and the body wave velocity $\alpha^{\prime}$ for the lossless cawe the complex propagation velocity $\boldsymbol{\alpha}$ can be written $\boldsymbol{\alpha}=\alpha^{\prime}\left(1-i Q_{a}{ }^{-1 / 2}\right.$ $\left.-Q_{a}{ }^{-2} / 4 \cdots \cdot\right)$ if there are no singularities in $k_{a}$. For $Q_{a}$ of 50 , a low value for earth materials, the real part of the propagation velocity is reduced by only $0.1 \%$ from the unattenuated value. This dispersion due to anelasticity will be ignored in the ensuing calculations.

The body velocities can also be written in terms of complex moduli and a real density,

$$
\begin{gathered}
\boldsymbol{\alpha}^{2}=[\mathbf{K}+(4 / 3) \mathbf{u}] / \rho \\
\hat{\beta}^{2}=\boldsymbol{u} / \rho
\end{gathered}
$$

Therefore

$Q_{\alpha}^{-1}=\left[K^{*}+(4 / 3) \mu^{*}\right] /[K+(4 / 3) \mu]$

$Q_{\beta}^{-1}=\mu^{* / \mu}$

By requiring the density to be real we ignore the possibility of losses due to imperfect inertia [Anderson and Archambean, 1964]. For an iso- tropic material the ratio of the body wave quality factors can be written

$$
\begin{array}{r}
Q_{\alpha}{ }^{-1} / Q_{\beta}{ }^{-1} \\
\quad=\left(\beta^{2} / \alpha^{2}\right)\left[K^{*}+(4 / 3) \mu^{*}\right] / \mu^{*}
\end{array}
$$

In Figure 1 this ratio is plotted against the square of the ratio of the body velocities for several $K^{*} / \mu^{*}$ ratios. In general, losses in pure

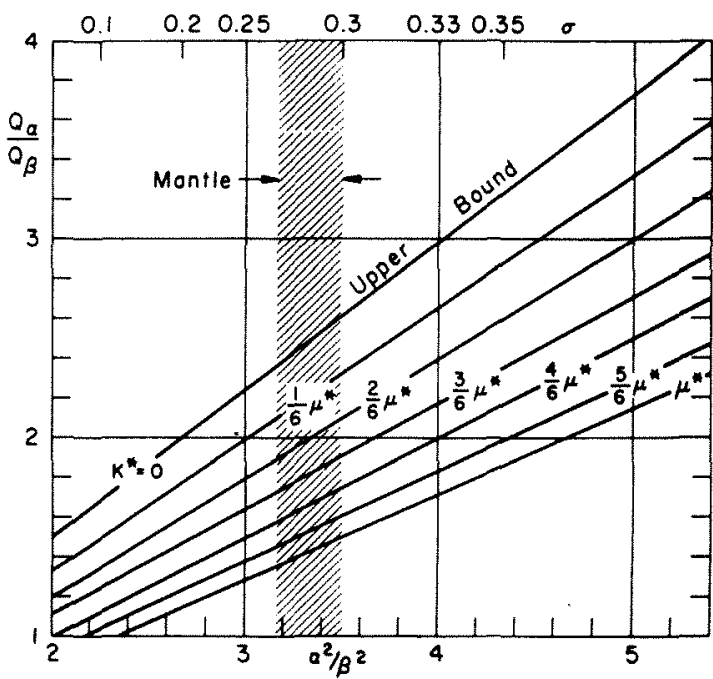

Fig. 1. Ratio of longitudinal to shear wave quality factors as a function of the square of the longitudinal to shear wave velocity ratio with complex compressibility as a parameter. 
compression are smaller than losses in shear. The curve for $K^{*}=0$ represents an upper bound for $Q_{a} / Q_{\beta}$ for isotropic materials. For a Poisson solid the longitudimal wave $Q$ for $K^{*}=$ 0 is $9 / 4$ the shear wave $Q$. For velocitics encountered in the mantle, $Q_{a}$ ranges from 2.35 to 2.60 times the shear wire $Q_{\beta}$. Korach and Anderson [1964] gave estimites of $Q_{3}$ of 600 , 200 , and 2200 respectively for the whole mintle, the upper mantle, and the lower mantle from an analysis of shear waves reflected from the core. The corresponding experiment camot be performed for $P$ waves, but the above alproach permits us to estimite $Q_{a}$ in the corresponding regions as 1500,500 , and 5500 .

In a homogeneous half-space Love waves do not exist and Rayleigh wave attenuation depends only on Poisson's ratio, $Q_{a}$, and $Q_{\beta}$. Press and Healy [1957] derived an expression relating: the absorption coefficients of Rayleigh waves to those of shear and compressional wires, which can be writton

$$
Q_{R}^{-1}=m Q_{\alpha}^{-1}+(1-m) Q_{\beta}^{-1}
$$

where $m$ is a function only of Poisson's ratio. Macdonald [1959] determined $m$ for a range of Poisson's ratios. From his tables we have computed $Q_{R} / Q_{\beta}$ versus $Q_{a} / Q_{\beta}$ with $\beta / \alpha$ as a param-

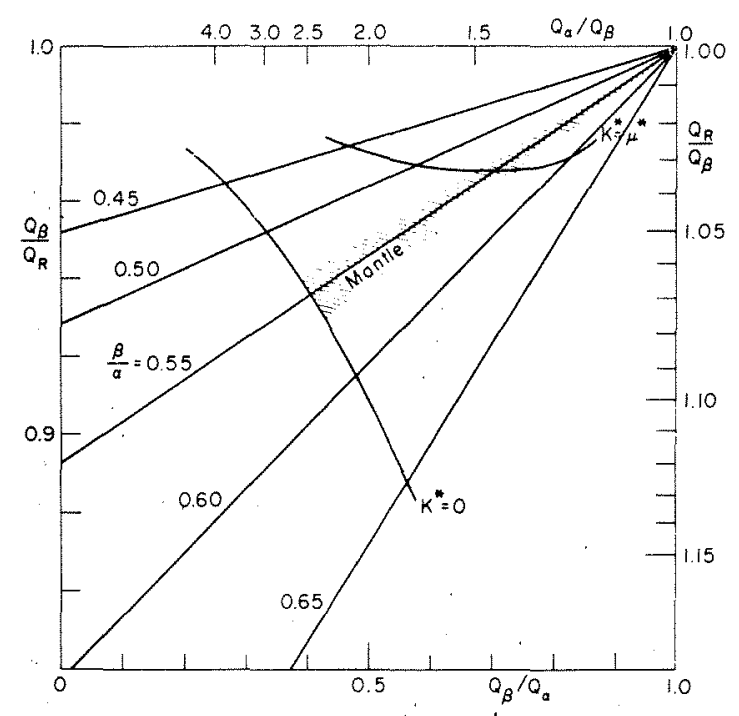

Fig. 2. Rayleigh wave quality factor $Q_{R}$ for a homogeneous half-space as a function of the ratio of body wave relocities and quality factors. The hatched region includes all values of $\beta / \alpha$ measured in the mantle. eter. The results are given in Figure 2. The shaded region includes all Poisson's ratios found in the mantle. The $Q$ for Rayleigh waves for a homogeneous half-space is roughly 3 to $7 \%$ greater than $Q$ for shear waves for reasonable assimptions about the $K^{*} / \mu^{*}$ ratio.

The above situations involve, to first order, nondispersive propagation. The theory of attenuation for dispersed waves has been treated by Anderson and Archambeau [1964]. For a dispersed wave train

$$
Q=c k / 2 \ell k^{*}
$$

All terms in this equation are, in general, frequency dependent. The complex phase velocity is

$$
\mathbf{c}=\omega / \mathbf{k}=c-\dot{i}^{*}
$$

Consider a layered wareguide composed of $N$ elastic layers of thickness $h_{l}$, compressional veloeity $\alpha_{l}$, shear velocity $\beta_{l}$, and density $\rho_{l}$. The diepersion relation

$$
c(\omega)=\omega / k=f\left(h_{l}, \alpha_{l}, \beta_{l}, \rho_{l}\right)
$$

cin be determined by a variety of techniques. Now introduce dissipation by making the velocities in each layer complex,

$$
\begin{aligned}
& \beta \rightarrow \beta-i \beta^{*} \\
& \alpha \rightarrow \alpha-i \alpha^{*}
\end{aligned}
$$

The wive number $k$ of the dispersed wave becomes complex and, for small attenuation,

$$
\begin{aligned}
\mathbf{k} & =k+i k^{*} \\
& =k+i\left[\sum_{l=1}^{k}\left(\frac{\partial k}{\partial \beta}\right)_{l} \beta_{l}^{*}+\sum_{i=1}^{v}\left(\frac{\partial k}{\partial \alpha}\right)_{l} \alpha_{l}^{*}\right]
\end{aligned}
$$

Therefore

$$
k^{*}=\sum\left(\frac{\beta}{2} \frac{\partial k}{\partial \beta}\right)_{l} Q_{\beta L}^{-1}+\sum\left(\frac{\alpha}{2} \frac{\partial k}{\partial \alpha}\right)_{l} Q_{\alpha l}{ }^{-1}
$$

The $Q$ of a dirpersed wave traveling on the sur. face of an inhomogeneous body may therefore be written

$$
\begin{aligned}
Q^{-1} & =2 \frac{U}{c}\left(\frac{k^{*}}{k}\right)_{\omega} \\
& =\frac{U}{c}\left[\sum\left(\frac{\beta_{l}}{k} \frac{\partial k}{\partial \beta_{l}}\right)_{\alpha \rho} Q_{\beta l}{ }^{-1}\right.
\end{aligned}
$$




$$
\begin{aligned}
& \left.+\sum\left(\frac{\alpha_{l}}{k} \frac{\partial k}{\partial \alpha_{l}}\right) Q_{\alpha i}^{-1}\right]_{\omega} \\
& =\frac{U}{c}\left[\sum\left(\frac{\partial Q^{-1}}{\partial Q_{\beta}^{-1}}\right)_{l} Q_{\beta l}^{-1}\right. \\
& \left.+\sum\left(\frac{\partial Q^{-1}}{\partial Q_{a}^{-1}}\right)_{l} Q_{a l}^{-1}\right]_{\omega} \\
& =\left[\sum\left(\frac{\partial Q^{-1}}{\partial Q_{\beta}^{-1}}\right)_{l} Q_{\beta l}^{-1}\right. \\
& \left.+\sum\left(\frac{\partial Q^{-1}}{\partial Q_{a}^{-1}}\right)_{l} Q_{\alpha l}^{-1}\right]_{l}
\end{aligned}
$$

Partial derivatives relilled to thowe in the above equation have becn computed for several realistic carth models by Anderson [196ta. b], Anderson and Archambere [1964], and Talieuchi et al. [1964].

For the present problem wo noe partial dorivatives calculated for a Gutomberg model carth by Tatienchi et al. [1064] and the following equations:

$$
\begin{aligned}
& Q_{l}{ }^{-1}=\sum_{l=1}^{N}\left(\frac{\beta_{l}}{c_{l}} \frac{\partial c_{L}}{\partial \beta_{l}}\right)_{t: p} Q_{\beta l}{ }^{-1} \\
& =\sum\left(\frac{\partial Q_{t}^{-1}}{\partial Q_{\beta t}^{-1}}\right)_{t_{i}} Q_{\beta t}^{-1} \\
& Q_{R}^{-1}=\sum_{l=1}^{x}\left(\frac{\alpha_{l}}{c_{n}} \frac{\partial c_{k}}{\partial \alpha_{l}}\right)_{l, p l a} Q_{a l}^{-1} \\
& +\sum_{l=1}^{N}\left(\frac{\beta_{l}}{c_{R}} \frac{\partial c_{R}}{\partial \beta_{l}}\right)_{l, \rho_{R}} Q_{\beta l}{ }^{-1} \\
& =\sum\left[\left(\frac{\partial Q_{R}^{-1}}{\partial Q_{\alpha x} l^{-1}}\right) Q_{\alpha l}{ }^{-1}\right. \\
& \left.+\left(\frac{\partial Q_{R}^{-1}}{\partial Q_{\beta l}{ }^{-1}}\right) Q_{\beta l}^{-1}\right]_{k}
\end{aligned}
$$

The subscript $l$ is the layer index; the subscripts $R, L, \alpha$, and $\beta$ associated with $Q$ identify the wave type. Other suberipts refor to quantities being held constant.

These functions are shown in Figure 3 for the

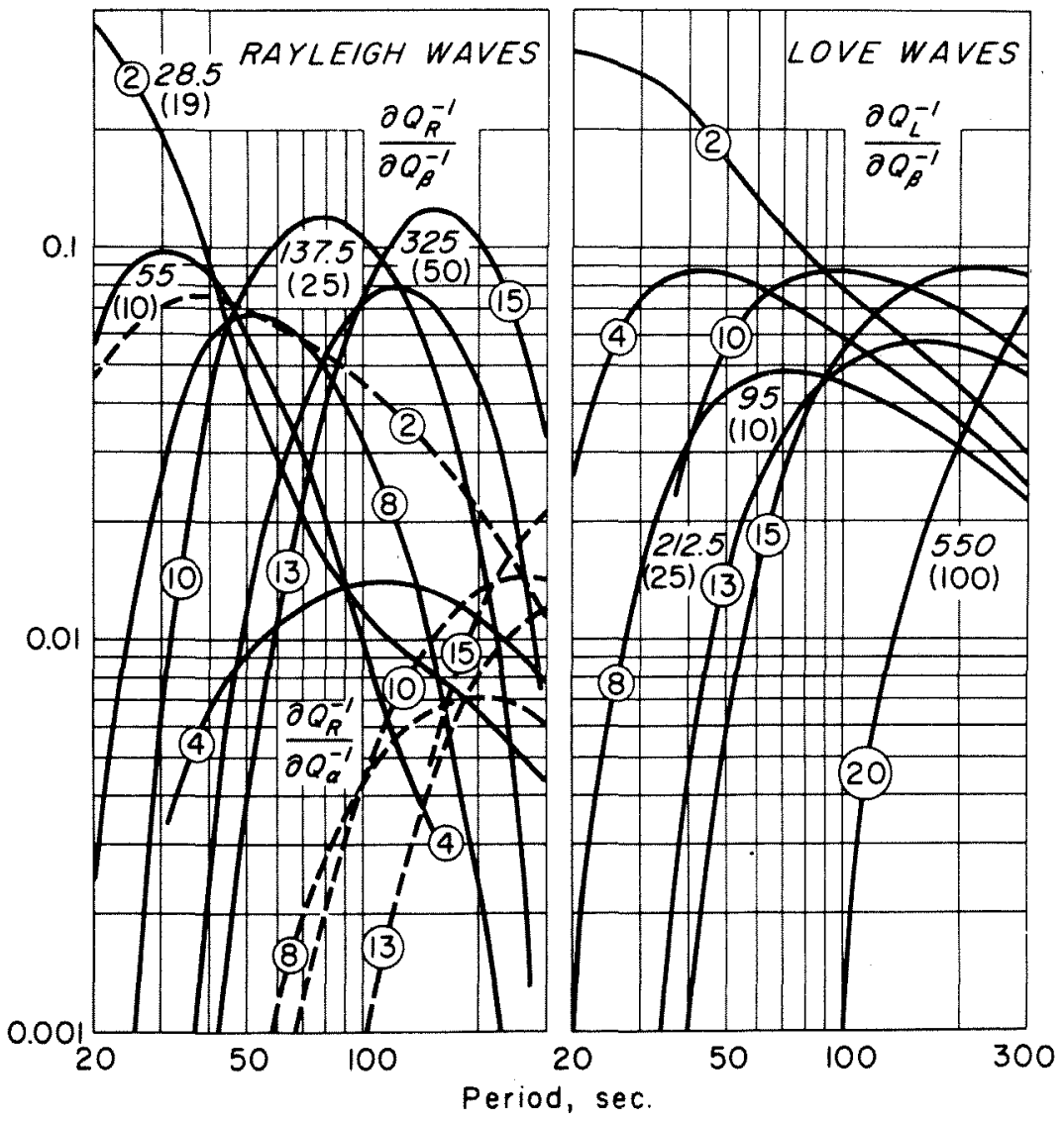

Fig. 3. Dimensionless curves showing the effect of $Q_{a}$ and $Q_{\beta}$ on $Q_{2}$ ind $Q_{R}$ for various layers in a Gutenberg-trpe continental earth model. Sec Dormon el al. [1960] for datie parameters of this model. The circled numbers on the curves are the layer indires. The depth and thickness of each layer are given adjacent to the curves. 


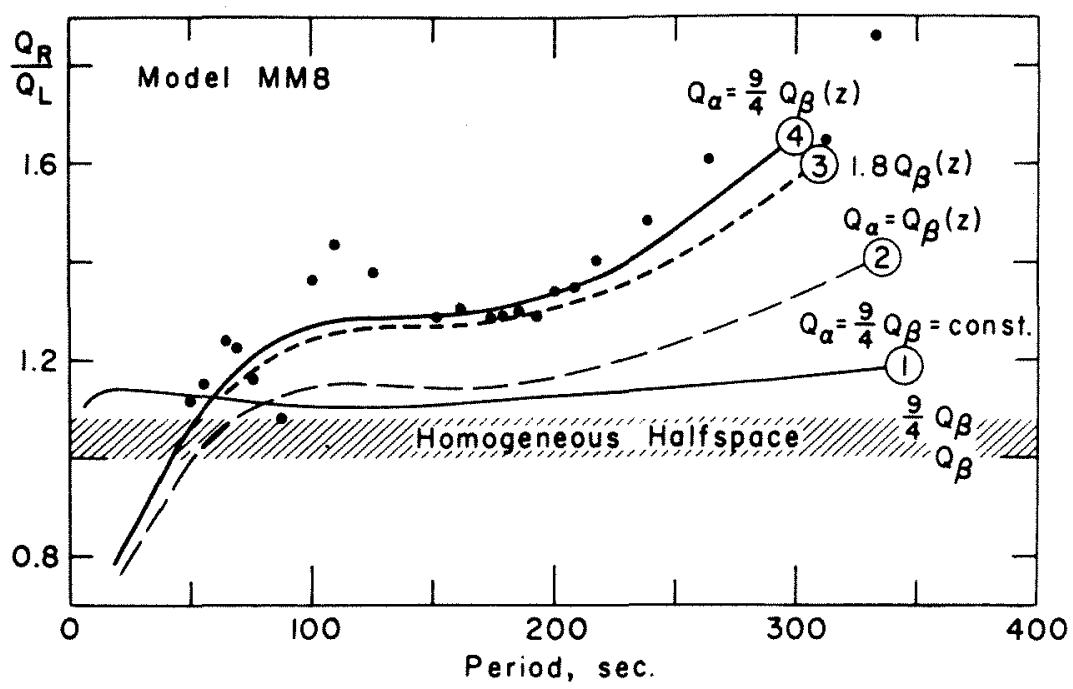

Fig. 4. The ratio $Q_{R} / Q_{L}$ for a constant $Q$ Gutenberg-type continental earth model (curvo 1), the heterogeneous $Q$ model MM8 for various $Q_{a} / Q_{\beta}$ ratios (curves 2,3 , and 4 ), and the ratio $Q_{R} / Q_{B}$ for a range of $Q_{a} / Q_{\beta}$ rntios for a homogeneous half-space (hatehed rogion). The data are from Tables 1 and 2.

period range 20 to 300 sec. Each curve indicates the effect of anelastic dissipation in a layer on Rayleigh or Love wave amplitudes as a function of period.

Love wave attenuation depends on the distribution of $\beta, \rho$, and $Q_{\beta}$. If $Q_{\beta}$ is constant with period and depth, $Q_{L}$ is frequency independent and equal to $Q_{\beta}$. $Q_{R}$ depends on the distribution of $\alpha, \beta, \rho, Q_{a}$, and $Q_{\beta}$ and is frequency dependent unless $Q_{\alpha}=Q_{\beta}=$ constant, in which case $Q_{R}=Q_{\beta}$ for all periods. These conclusions follow from the properties of the partial deriviltives in (17).

In Figure 4 (curve 1) we show the variation of $Q_{R} / Q_{L}$ versus period for a Gutenberg-type earth model in which $Q_{a}$ and $Q_{\beta}$ are constant with depth and $Q_{a}$ is equal to $(9 / 4) Q_{\beta}$. For this case $Q_{R}$ is roughly 10 to $16 \%$ greater than $Q_{L}$ and exhibits a mild frequency dependence.

Models for $Q$. A satisfactory $Q$ distribution for the earth must explain the observed frequency dependence of both Love and Rayleigh waves and be consistent with body wave and laboratory results. The latter contraints are mild because of the paucity of data.

We have designed several $Q$ models for the upper part of the earth, taking as starting values the $Q$ structure proposed by Anderson and Archambeau [1964] and as a constraint the crustal $Q_{a}$ of 450 determined by Press [1964].
We further assume isotropy of $Q$; i.e., $Q_{\beta}$ for Love waves is the same as $Q_{\beta}$ for Rayleigh waves. For the models considered we assume that the intrinsic $Q$ at a given depth is independent of frequency. None of these constraints and assumptions are necessary for the solution of the problem, but they are reasonable and we have been able to find satisfactory models without violating these basic guidelines. The most severe limitation of our present analysis is the lack of shorter-period data which would allow us to make an independent determination of the crustal $Q$.

Table 1 gives the parameters of a nodel which we have designated MM8. The results of $Q_{R}$ and $Q_{L}$ versus period are plotted in Figure 5 for various assumptions concerning the $Q_{a} / Q_{\beta}$ ratio. The curve labeled $Q_{a}=2.25 Q_{\beta}$ is an upper bound for Rayleigh wives for an isotropic Poisson solid. We consider the fit of this curve to the experimental data to be satisfactory. Thus the assumption that $K^{*}=0$, i.e. no losses in pure compression, seems to be a good one, although experimental error will allow values as high as $K^{*}=\mu^{*} / 2$. We do not consider the data good enough to permit us to determine the attenuation of longitudinal waves directly, but we can safely say that $2.5 Q_{\beta}>Q_{a}>1.8 Q_{\beta}$ for the elastic and $Q_{\beta}$ model chosen. This seems to rule out major nonadiabatic or thermal con- 


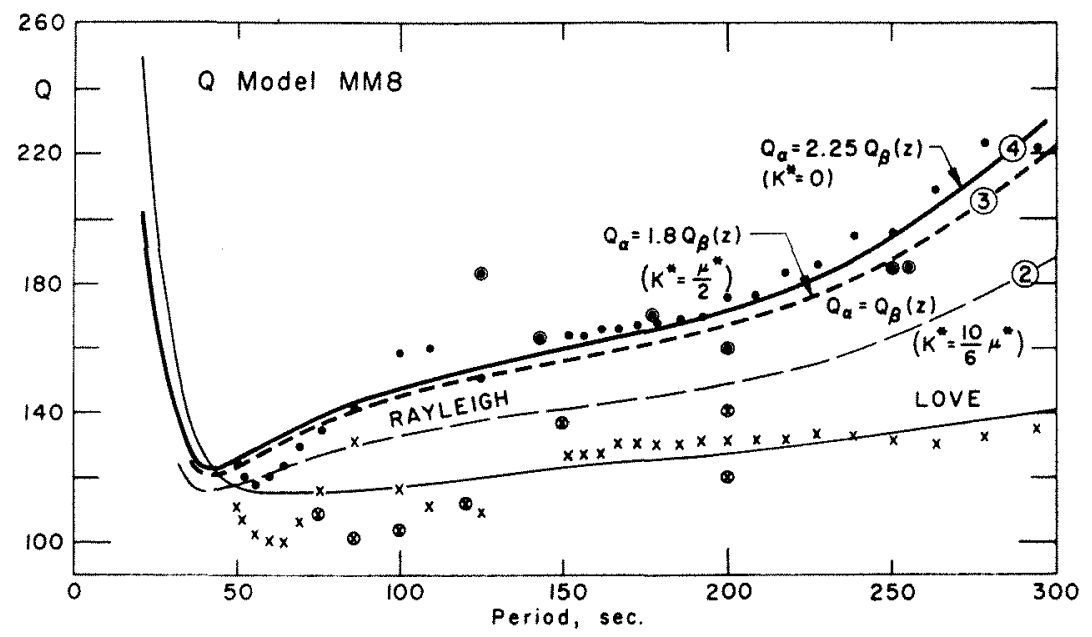

Fig. 5. Attenuation data from this study for Love $(\mathrm{X})$ and Rayleigh (.) waves. The circled data are from Savarensky et al. [1961], Euing and Press [1954], Båth and Lopez Arroyo [1962], and Satô [1958]. The curves are theorntical results for model MM8.

duction losses. The $Q_{a} / Q_{\beta}$ or $Q_{R} / Q_{L}$, ratio is of great importance in isolating the loss mechanism.

The $Q_{R} / Q_{L}$ ratio, both experimental and theoretical, is plotted in Figure 4. Again we have the strong suggestion that $K^{*} \approx 0$ in the earth, but more data of this type are definitely required to make a botier determination of $K^{*}$ * or $Q_{a}$.

Shorter-period data, between 10 and $40 \mathrm{sec}$, are required in order to determine the depth of the rapid decrease in $Q$ that seems to take place in the lower crust or upper mantle. It would be very interesting to see if this $Q$ discontinuity is near the depth of the Mohorovicic $(M)$ discontinuity or appreciably deeper. The upper-mantle $Q$ discontinuity is more closely related to questions of isostasy, mountain building, continental

TABLE 3. Model MM8

\begin{tabular}{rrr}
\hline $\begin{array}{r}D, \\
\mathrm{~km}\end{array}$ & \multicolumn{1}{c}{$Q_{\beta}$} & \multicolumn{1}{c}{$Q_{\alpha}$} \\
\hline 38 & 450 & 1012.5 \\
22 & 60 & 135.0 \\
10 & 80 & 180.0 \\
55 & 100 & 225.0 \\
375 & 150 & 337.5 \\
100 & 180 & 405.0 \\
100 & 250 & 562.5 \\
100 & 450 & 1012.5 \\
100 & 500 & 1125.0 \\
100 & 600 & 1350.0 \\
\hline
\end{tabular}

drift, and phase changes than is the $M$ discontinuity itself. We hope to answer this question in the near future.

Our present data are most sensitive to paramcters in the upper $400 \mathrm{~km}$ of the mantle, although we have taken our models deeper. The $Q_{\beta}$ varies from about 60 beneath the 'crust' to about 150 at $400 \mathrm{~km}$. The corresponding $Q_{a}$ variation, with a large uncertainty, would be from about 135 to 340 . Although our uncertainty in $Q$ below $400 \mathrm{~km}$ is great, model MM8 gives an average $Q_{\beta}$ of 144 for the upper 600 $\mathrm{km}$. This can be compared with the estimates of 185, 151, and 200 given by Anderson and Kovach [1964] and Kovach and Anderson [1964].

Fedotov [1963] evaluated the absorption coefficient of 0.2 - to 0.5 -cps shear waves in the earth's crust and upper mantle in the vicinity

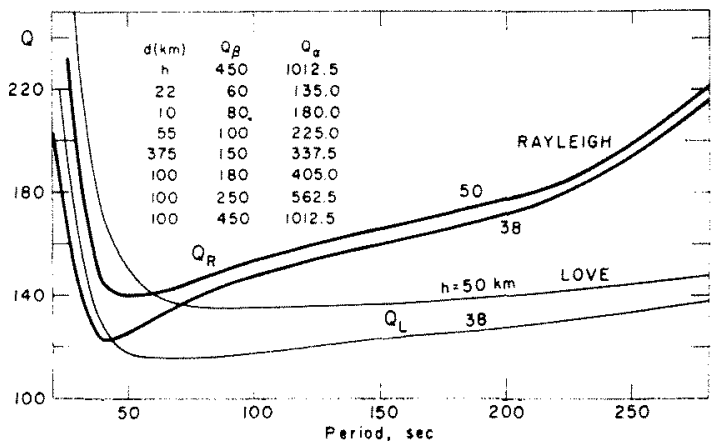

Fig. 6. The effect of crustal thickness on $Q$ of Love and Rayleigh waves. 
of the southern Kurile Islands. From his values for $K^{*}$ and $\beta$ we find values for $Q$ of roughly $400 \pm 200$ for the crust, $80 \pm 30$ at $70 \mathrm{~km}$, and $130 \pm 60$ at $120 \mathrm{~km}$. Model MIMS has values of $Q_{\beta}$ of 450,80 to 100 , and 100 to $150 \mathrm{in}$ the corresponding regions. The large imeertaintios assigned to Fedotor's values are due primarily to uncertainties in period.

The effect of a change in crustal thickness is shown in Figure 6, where model MML, which has a $38-\mathrm{km}$ high $Q$ cmst, is compared with a model that is identical in all respects exeept that the high $Q$ crust is increased to $50 \mathrm{~km}$, the $M$ discontinuity remaining at $38 \mathrm{~km}$. In both cases there is a crossover of $Q_{R}$ with $Q_{L}$ at the shorter periods. The crossover takes place at 43 sec for the thinner crust and at 60 sec for the $50-\mathrm{km}$ thick $Q$ crust. A rapid increase in $Q_{L}$ occurs at a slightly greater period and in $Q_{R}$ at a slightly shorter period than the crossover period. The location of the crossover, or of the rapid increase in $Q$ if only $Q_{n}$ or $Q_{n}$ is measured, is diagnostic of the depth of the $Q$ discontinuity. The effects of scattering at the shorter periods and of the core at longer periods will be considered in a separate paper. With our present resolution, they do not affect data in the period range considered.

Acknowledgment. This researeh was supported by contract AF-49(638)-1337 of the Air Force Office of Scientific Research as part of the Advanced Research Projects Agency project Veli.

\section{REFERENCES}

Alexander, S. A., Surface wave propagation in the western United States, thesis, California Institute of Technology, Pasadena, 1963.

Anderson, Don L., Recent evidence concerning the structure and composition of the earth's mantle, Physics and Chemistry of the Earth, chap. 1, pp. 1-129, Pergamon Press, New York and London, $1964 a$.

Anderson, Don L., Universal dispersion tables, 1, Love waves across oceans and continents on a spherial eath, Bull. Seismol. Soc. Am., 5q, 681$726,1964 b$.

Anderson, Don L., and C. B. Archambeat, The anelasticity of the earth, J. Geophys. Res., 69, 2071-2084, 1964.

Anderson, Don L., and R. I. Fovach, Attenuation in the mantle and rigidity of the core from multiply reflected corc phases, Proc. Natl. Acad. Sci. U.S., 51, 168-172, 1964.

Bith, M., and A. Loncz Arroyo, Attenuation and dispersion of $G$ waves, $J$. Geophlys. Res., $b^{r}$, 1933-1942, 1962.

Dorman, J., M. Ewing, and J. Oliver, Study of shear-relocity distribution in the upper mantle by mantle Ravleigh waves, Bull. Seismol. Soc. A im., 50, 87-115, 1960 .

Ewing, M., and F. Press, Mantle Rayleigh waves from the Kamchatlia earthquake of November 4, 1952, Bull. Seismol. Soc. Am., 44, 471-479, 1954.

Fedotov, S. A., The absorption of transverse seismic waves in the upper mantle and energy classification of near earthquakes of intermediate focal depth, Izv. Alad. Nauk SSSR, Ser. Geofiz., $509-520,1963$.

Kovach, R. L., and Don I. Anderson, Attemuation of shear wares in the upper and lower mantle, in press, Bull. Seismol. Soc. Am., 1964.

Macdonald, J. R., Rayleigh-wave dissipation functions in low-loss media, Geophys. J., 2, 132-135, 1959.

Press, F., Seismic wave attenuation in the crust, J. Geophys. Res., 69, 4417-4418, 1964.

Press, F., and J. Healy, Absorption of Rayleigh waves in low-lows media, J. Appl. Phys., 28, $1323-1325,1957$.

Sato, Y., Attenuation, dispersion and the wave guide of the $G$ wave, Bull. Seismol. Soc. Am., $48,231-251,1958$.

Sulvirensky, E. F., I. I. Popov, and A. P. Lazareva, Observations of long periods waves of the Chilean varthquake of 1960, Izv. Alad. Nauk SSSR, Ser. Geofiz., 744-748, 1961.

Takeuchi, H., J. Dorman, and M. Saito, Partial derivatives of surface wave phase velocity with respect to physical parameter changes within the earth, J. Geophys. Res., 69, 3429-3442, 1964.

Toksöz, M. N., and A. Ben-Menahem, Velocities of mantle Love and Rayleigh waves over multiple paths, Bull. Seismol. Soc. Am., 53, 741-764, 1963.

(Manuscript received October 6, 1964.) 